\title{
THE ACCURACY OF TWO-DIMENSIONAL SACCADES IN THE ABSENCE OF CONTINUING RETINAL STIMULATION
}

\author{
Melvin K. Komoda, ' Leon Festinger and Johniathay Sherry \\ New School for Social Research, New York. NY 10011, U.S.A.
}

(Receited 2 February 1977; in retised form 18 . Warch 1977)

The ability of observers to execute saccades in the dark to previously visible targets has been demonstrated by Becker and Fuchs (1969) and Becker and Klein (1973). Becker and Fuchs report the occurrence of horizontal saccades when a target, $40^{3}$ away from fixation. was extinguished $350 \mathrm{msec}$ prior to the initiation of the saccade. Becker and Klein report that observers, after practicing horizontal saccades to targets were able to continue making goal-directed saccades in total darkness. The accuracy of these saccades, however, decreased with time. These results imply that continuing retinal stimulation is not necessary for the execution of goal-directed saccades. Rather, the saccadic control system appears able to utilize some sort of stored information. The present experiment assessed the accuracy with which the saccadic control system could execute, from memory alone, a consecutive series of two-dimensional saccades to targets randomly located in the fronto-paralle! plane.

\section{METHOD}

\section{Obsercers}

Three naive observers participated in the experiment They all had normal visual acuity and binocular coordination.

\section{Apparatus}

Computer-generated target patterns were displayed on a Hewlett-Packard 1310 oscilloscope equipped with a very rapidly decaying phosphor (P15). thus insuring the absence of any persisting physical images. The face of the oscilloscope was located $1 \mathrm{~m}$ from the observer and provided a $\pm 8^{\prime}$ visual field horizontally and vertically.

Eye movements were measured using the double-Purkinje-image "eye tracker" developed by Cornsweet and Crane (1973). An observer's head was held fixed by using a biteboard and headrest and, movements of the right eye were measured with the left eye occluded. Both horizontal and vertical analogue outputs of the eye tracker indicating the position of the eye were sampled every $2 \mathrm{msec}$ and digitized with a resolution equivalent to $0.5^{\prime}$ by the computer system; this is lower than the eye tracker's noise level of 2'. Information about the target pattern and the corresponding eye movement data were stored on magnetic tape for subsequent analysis.

\section{Procedure}

The experiment consisted of three $2 \cdot$ hr sessions. The entire first session was devoted to the calibration of the

\footnotetext{
${ }^{1}$ Present address: Department of Psychology, Concordia University. Montreal, Quebec. Canada, H3G $1 \mathrm{M} 8$.
}

observer's eve movements using the procedure described in Festinger. Sedgwick and Holtzman (1976).

The target patterns consisted of an initial fixation point and three discriminably different targets. Targets 1,2 and 3 were each located at any one of 49 possible locations specified by the combination of seven horizontal and seven vertical values. For each trial, these locations were randomly chosen with the constraints that the targets could appear neither at the center of the matrix nor at identical locations. Adjacent locations in the matrix along the horizontal or vertical axes were separated by $2^{\circ}$. The observer's task was successively to fixate the three targets in the prescribed order. Target 1 . Target 2 and finally Target 3.

At the start of each trial the fixation point was displayed in the center of the visual field in an otherwise dark room. The three targets, together with the fixation point, then appeared. Following the appearance of the targets. any eye movement greater than $30^{\circ}$ triggered one of the following three conditions: (1) the "All Off" condition in which the initial fxation point and all targets were extinguished; (2) the "Fixation On" condition in which the targets were extinguished but the fixation point remained visible; (3) the "All On" condition in which all stimuli remained visible.

The observers were instructed to complete the task by attempting to fixate, in the prescribed order, the remembered locations of the targets. They were encouraged to take enough time and to be accurate. The three conditions were presented in random order. Each trial lasted $4 \mathrm{sec}$.

All observers were given a number of "All On" practice trials prior to the experimental trials. Experimental trials were presented in blocks of 10-15 trials, a block of trials lasting approximately $2 \mathrm{~min}$. The observers had a short rest between blocks of trials.

\section{RESULTS AND DISCUSSION}

The accuracy of a saccade was assessed by comparing both the magnitude and direction of the eye movement vector to the respective components of the target vector whose origin was the location of the prior target and whose termination was the location of the target to which the saccade was directed. For example, the accuracy of a saccade to Target 2 was assessed by comparing the eye movement vector of the saccade to the target vector that extended from the location of Target 1 to the location of Target 2.

Inasmuch as the comparison of the eye movement and target vectors could reveal highly accurate saccades (i.e. small magnitude and directional errors) despite systematic errors of fixation, the fixation error to a target was also computed. The fixation error was specified as the Euclidian distance between the pos1231 
Table 1. The accuracy of saccades to successive targets

\begin{tabular}{|c|c|c|c|c|c|c|c|}
\hline \multirow{3}{*}{ Condition } & \multirow{3}{*}{ Saceade to: } & \multicolumn{6}{|c|}{ TYPE OF ERROR } \\
\hline & & \multicolumn{2}{|c|}{$\begin{array}{l}\text { Magni tude of Saccade } \\
\text { (maan algebraic error) }\end{array}$} & \multicolumn{2}{|c|}{$\begin{array}{l}\text { Direction of Saccade } \\
\text { (mean dbsolute error) }\end{array}$} & \multicolumn{2}{|c|}{$\begin{array}{l}\text { Fixation of Target } \\
\text { (mean absolvte error) }\end{array}$} \\
\hline & & Mean & (S.D.) & Mean & $(s .0)$. & Mean & (5.0) \\
\hline \multirow{3}{*}{$\begin{array}{l}\text { "Targets off" } \\
\text { (Average N per } \\
\text { Observer = 143.0) }\end{array}$} & Target 1 & .0 .42 & $(0.72)$ & 3.33 & $\{2.64\}$ & 0.72 & $(0.59)$ \\
\hline & Target 2 & 0.37 & $(1.86)$ & 7.67 & $(7.19)$ & 2.05 & $(1.26)$ \\
\hline & Target 3 & 0.87 & $(2.39)$ & 8.77 & $(7.46)$ & 2.25 & $(1.1 T)$ \\
\hline \multirow{3}{*}{$\begin{array}{l}\text { "Targets On" } \\
\text { (Average N per } \\
\text { observer }=76.7 \text { ) }\end{array}$} & Target 1 & -0.42 & $(0.66)$ & 2.85 & $(2.61)$ & $0.6 T$ & $(0.51)$ \\
\hline & Target 2 & -0.13 & $(0.69)$ & 2.21 & $(2.15)$ & 0.56 & $(0.34)$ \\
\hline & Target 3 & 0.06 & $(0.51)$ & 1.77 & $(1.78)$ & 0.51 & $(0.35)$ \\
\hline
\end{tabular}

ition of the eye at the end of a saccade and the location of the appropriate target.

The analysis of each observer's data showed that the results for the "All Off" and "Fixation On" conditions were similar. Consequently, the data for these two conditions have been combined and will be referred to as the "Targets Off" condition.

The results on the accuracy of the saccades are presented in Table 1. Since all three observers showed similar results, the table shows the data averaged for the three observers. The data on magnitude errors of saccades are mean algebraic errors. The data on directional and fixation errors are mean absolute errors. The columns headed (S.D.) present the average "within observer" standard deviation.

In order to provide a context for evaluating the accuracy of the saccades to Targets 2 and 3 in the "Targets Off" condition, it should be stated that the mean magnitudes of target vectors was $8.53^{\circ}$ from Target 1 to Target 2 , and $8.14^{\circ}$ from Target 2 to Target 3 . The mean magnitudes of the corrresponding target vectors in the "Targets On" condition were 8.08 and $8.43^{\circ}$, respectively. It is clear from the data that the observers were able to execute two consecutive saccades from memory with reasonable accuracy. The accuracy of the saccade to Target 1 is, of course, comparable in the "Targets On" and "Targets Off" conditions, since the execution of the first saccade began before targets were extinguished and the observer did not know which condition he would encounter.

It is also clear from Table 1 that there is a decrease in the accuracy of saccades executed in the absence of continued retinal stimulation. Saccades to Targets 2 and 3 in the "Targets Off" condition have larger magnitude, direction and fixation errors than the corresponding saccades in the "Targets On" condition. The lower accuracy of these saccades in the "Targets Off" condition is also indicated by the larger standard deviations associated with all three error measures. It is also worth noting that. in the absence of retinal stimulation, the magnitude and direction errors for Target 3 tend to be larger and more variable than for Target 2. This finding is consistent with the report by Becker and Klein (1973).

We have interpreted the data as showing that a consecutive series of two saccades can be executed with reasonable accuracy on the basis of remembered target locations. It is possible, however, to contend that, perhaps, all the saccades were preprogrammed while the targets were still visible. Such a contention does not seem plausible, however. in view of the intersaccadic latencies involved. Since we urged the observers to take their time and to emphasize accuracy, these latencies are quite large. The intersaccadic latencies for the saccade from Target 1 to Target : averaged $769 \mathrm{msec}$ for "Targets Off" and $667 \mathrm{msec}$ for "Targets On". The comparable values for the latency of the saccade between Target 2 and Target 3 are 808 and $738 \mathrm{msec}$. It seems more likely that the saccades were indeed, programmed on the basis of memory.

Acknowledgements-This research on which this article is based was supported by Grant Number MH-16327 from the National Institute of Mental Health to Leon Festinger.

\section{REFERENCES}

Becker W. and Fuchs A. F. (1969) Further properties of the human saccadic system: eye movements and correc. tion saccades with and without visual fixation points Vision Res. 9, 1247-1257.

Becker W. and Klein H. M. (1973) Accuracy of saccadis eye movements and maintenance of eccentric eye pos. ition in the dark. Vision Res. 13, 1021-1034.

Cornsweet T. N. and Crane H. D. (1973) Accurate two dimensional eye tracker using first and fourth Purkinj images. J. opt. Soc. Am. 63, 921-928.

Festinger L. Sedgwick H. A. and Holtzman J. D. 11976 Visual perception during smooth pursuit eye move ments. Vision Res. 16, 1377-1386. 